\section{Una experiencia de curricularización de la extensión en Bioingeniería}

\section{Fernando Sassetti}

fsassetti@ioingenieria.edu.ar

Marisol Perassi

mperassi@bioingenieria.edu.ar

\section{Diego Kadur}

dkadur@bioingenieria.edu.ar

Docentes investigadores de la Facultad de Ingeniería. Universidad Nacional de Entre Ríos, Argentina.

\author{
Javier Billordo \\ Becario de proyecto de innovación \\ pedagógica de la Universidad \\ Nacional de entre Ríos. \\ javierbillordo12@gmail.com
}

Integración de la docencia y la extensión /

Intervenciones

RECEPCIÓN: 24/06/16

ACEPTACIÓN FINAL: 04/10/16

\section{Resumen}

Este artículo presenta la experiencia de curricularización de la extensión universitaria implementada en la asignatura "Organización de los sistemas de salud" de la carrera de Bioingeniería de la Facultad de Ingeniería de la Universidad Nacional de Entre Ríos durante los años 2014 y 2015. La metodología desarrollada para integrar las actividades de extensión y de enseñanza se basa en la estrategia de Aprendizaje Basado en Proyectos. A modo de evaluación de la misma, se incluyen las valoraciones de los estudiantes respecto de cómo vivenciaron una forma de aprendizaje basada en lo experiencial. Los mismos reconocen que las acciones de articulación docencia-extensión son de central importancia para la formación crítica y contextualizada como futuros profesionales.
Palabras-clave

- Extensión universitaria

- Enseñanza superior

- Aprendizaje activo

\section{Resumo}

Este artigo apresenta a experiência de curricularização da extensão universitária implementada na disciplina "Organización de los sistemas de salud", do curso de Bioengenharia da Facultade de Engenharia da Universidad Nacional de Entre Ríos durante os anos de 2014 e 2015. A metodologia desenvolvida para integrar atividades de extensão e ensino baseia-se na estratégia de Aprendizagem Baseada em Projetos. Como avaliação se incluem as opiniões dos estudantes a respeito de como vivenciaram uma forma de aprendizagem baseada no experiencial. Eles reconhecem que as ações de articular docência-extensão são de importância central para a formação crítica e contextualizada como futuros profissionais.

Palavras-chave

- Extensão universitária

- Ensino superior

- Aprendizagem ativa

\section{Para citación de este artículo}

Sassetti, F.; Perassi, M.; Kadur, D. y Billordo, J. (2016). Una experiencia de curricularización de la extensión en Bioingeniería. En Revista +E versión digital, (6), pp. 396-403. Santa Fe, Argentina: Ediciones UNL. 


\section{Introducción}

Este artículo describe la experiencia de curricularización de la extensión que comenzó en el año 2014 en la asignatura "Organización de los sistemas de salud". La asignatura es electiva, cuatrimestral con una carga horaria semanal de 6 horas y pertenece al $5^{\circ}$ año de la carrera de Bioingeniería.

En el desarrollo del cursado se presentan a los estudiantes problemas relacionados con el desarrollo de su carrera profesional en organizaciones dedicadas a la atención de la salud del sector público y privado.

Desde 2007 la cátedra desarrolla proyectos de extensión universitaria y desde 2013 implementa como estrategia didáctica en el desarrollo de toda la asignatura el Aprendizaje Basado en Proyectos (ABP). Ambas líneas de trabajo de la cátedra encuentran su articulación en el año 2014 con la elaboración de un Proyecto de Curricularización de la extensión universitaria, ${ }^{1}$ a partir del cual se comienza la integración entre las actividades de enseñanza y de extensión que continúa hasta hoy.

Dicho Proyecto recupera y articula entonces, por un lado, la experiencia acumulada de la cátedra en cuanto a actividades de extensión universitaria, y por otro lado, ideas y propuestas pedagógicas desarrolladas en diferentes instancias de formación docente organizadas desde la Secretaría Académica y la Asesoría Pedagógica de la Facultad. En diversos talleres, cursos y jornadas, destinadas a docentes se abordaron, por ejemplo, las estrategias de aprendizaje activo como el Aprendizaje basado en problemas o proyectos.

En el presente artículo se intentará responder a preguntas respecto de la integración extensión-enseñanza, tales como: ¿de qué manera lograr una articulación entre extensión y enseñanza en

1) La convocatoria para la presentación de Proyectos de curricularización de la extensión se abrió por primera vez en la UNER en el año 2014. El Proyecto presentado por la cátedra se la universidad? ¿Es válida la metodología de aprendizaje activo basada en proyectos para dicha integración? ¿Cómo evaluar el proceso y la experiencia realizada? ¿Cómo lo valoran los estudiantes y los docentes?

\section{Fundamentos para la curricularización de la extensión}

Los estudios realizados desde el campo de la psicología cognitiva han identificado ya hace tiempo dos grandes deficiencias respecto de los resultados del modelo tradicional de educación: el "conocimiento frágil" y el "pensamiento pobre". El primero aparece cuando "los estudiantes no recuerdan, no comprenden o no usan activamente gran parte de lo que supuestamente han aprendido" y el segundo cuando "Ios estudiantes no saben pensar valiéndose de lo que saben" (Perkins, 1997:32).

Para contrarrestar estos efectos, distintas investigaciones resaltan la importancia del uso activo del conocimiento para favorecer su comprensión:

"el conocimiento debe ser construido activamente por los alumnos (...). No es posible 'transmitir' simplemente a los alumnos los secretos de la pericia. Esto no significa que la información proporcionada por docentes y textos no sea importante. Sin embargo, sugiere que los alumnos deben tener la posibilidad de usar activamente esa información por sí mismos y experimentar sus efectos sobre su propio desempeño. Si no tienen la posibilidad de usar la nueva información para lograr objetivos específicos, los alumnos a menudo aprenden hechos que sólo se pueden recordar en contextos específicos y que, de lo contrario, permanecen 'inertes'". (Whitehead, 1929; citado en Perkins, 1997) 
"La información no se usa para resolver problemas" (Brandsford y Vye, en Resnick y Klpfer, 1996:299).

Esto es particularmente válido en la universidad, donde en muchos casos aún se reproducen prácticas tradicionales de enseñanza y de evaluación que no aportan a la comprensión del conocimiento sino que terminan favoreciendo un estudio meramente memorístico. Algunas de las estrategias didácticas que favorecen el uso activo de los conocimientos son las denominadas "metodologías de aprendizaje activo" (Mastache, 2007), entre las que se encuentra el ABP.

Tanto el ABP como otras estrategias de aprendizaje activo suponen: reproducción de situaciones reales, significatividad social y subjetiva, articulación teoría-práctica, consideración de las incertidumbres, ambigüedades y contradicciones e integración disciplinar (Mastache, 2007). Además, implican cambio de los roles tradicionales de docente y estudiante, toma de decisiones y fundamentación por parte de los estudiantes (ITESM, 2000) y trabajo en grupo (Camilloni, 2010).

Este tipo de estrategias favorece lo que Bain denominó:

"[un] entorno para el aprendizaje crítico natural", [en el cual] "las personas aprenden enfrentándose a problemas importantes, atractivos o intrigantes, a tareas auténticas que les plantearán un desafío a la hora de tratar con ideas nuevas, recapacitar sus supuestos y examinar sus modelos mentales de la realidad. Son condiciones exigentes pero útiles, en las que los estudiantes experimentan una situación de control sobre su propia educación; trabajan en colaboración con otros”. (2006:29)

Como estrategia didáctica, el ABP no tiene un origen reciente ya que se remonta al inicio del siglo XX.

"El trabajo con proyectos fue propuesto por William Kilpatrick en 1917 a partir de la influencia recibida de John Dewey, creador de la escuela activa, quien sostenía que era necesario aprender haciendo" (Anijovich, 2010:93).

Por proyecto se entiende una propuesta de solución a determinado problema que, además, implica algún grado de diseño y puede incluir o no su implementación. Como resultado, genera productos diversos: tesinas, diseño de productos o dispositivos, modelos, programas informáticos, elaboración de propuestas, etc. (Mastache, 2007). Es una estrategia prolongada en el tiempo que suele extenderse a lo largo de todo un curso e incluye en fases y actividades diversas (planificación, desarrollo, evaluación, comunicación, etc.). Asimismo, es habitual que el proyecto implique vínculos de los estudiantes con otros actores, otras organizaciones, otros profesionales de la sociedad, superando de este modo el habitual trabajo pedagógico reducido el espacio áulico.
A su vez, esta estrategia didáctica puede ser concebida como un modo de incluir la educación experiencial en el curriculum universitario, en tanto se aproxima al denominado "aprendizajeservicio", en el cual "los estudiantes aplican sus habilidades y conocimientos académicos y profesionales específicos para satisfacer necesidades sociales reales en respuesta a requerimientos explícitos de la comunidad" (Camilloni, 2013:17). Por su parte, desde la perspectiva de curricularización de la extensión, se concibe la extensión universitaria como:

"un proceso dialógico y bidireccional, que busca la colaboración entre actores universitarios y actores sociales, en un pie de igualdad, dialogando y combinando sus respectivos saberes al servicio de objetivos socialmente valiosos, dando prioridad a los problemas de los sectores más postergados". (Citado en Ramírez y Gantus, 2012)

Las acciones de integración de la extensión al curriculum universitario permiten, en efecto, discutir con varias ideas y prácticas que actualmente parecen encontrarse naturalizadas en la universidad.

Una de ellas es la idea de que la enseñanza y el aprendizaje en la universidad deben darse al interior de las aulas. "Debemos poner en crisis esta idea de que todo lo que debiéramos enseñar y aprender en la Universidad circule entre estas cuatro paredes" (Cecchi, 2015:30).

El aprendizaje que se genera al integrar la extensión a la enseñanza es un aprendizaje situado, se aprende participando en prácticas con la comunidad, se aprende haciendo en contextos vinculados con las futuras prácticas profesionales, lo cual supone el desarrollo de competencias concebidas como saberes en acción, como praxis que articula conocimiento y práctica (Macchiarola, 2010). Además, la concepción de conocimiento misma se transforma, en tanto se conjugan diversos saberes: académicos, científicos, humanísticos, técnicos, cotidianos, prácticos, populares, etc., los cuales pueden enriquecerse en su interacción (Macchiarola, 2010). También se transforma la idea del curriculum universitario, ya que es superadora de la racionalidad técnica que postula que primero debe transmitirse la teoría y luego procurarse su aplicación en contextos prácticos, reemplazándola por una racionalidad práctica y crítica de reflexión sobre y para la acción transformadora (ídem).

\section{Metodología}

La metodología que implementa la cátedra consiste principalmente de un trabajo a partir del cual los estudiantes —en grupos y a lo largo de todo el cursado-identifican y analizan problemáticas propias de las organizaciones y de las poblaciones en las que 
estas están insertas y formulan un proyecto para su abordaje. El trabajo supone una diversificación de los espacios de enseñanza y aprendizaje y, en este sentido, los docentes y estudiantes realizan diversas actividades tales como: recorrer diferentes barrios, visitar organizaciones relacionadas directa o indirectamente con la salud pública (centros de salud, escuelas, comisiones vecinales, centros comunitarios, etc.), efectuar consultas y entrevistas a los vecinos y trabajadores del sistema de salud (agentes sanitarios, psicólogos, trabajadores sociales, administrativos, médicos, etc.), organizar reuniones con diferentes actores sociales relacionados con el abordaje de la salud (charlas, jornadas de discusión, etc.), participar en eventos académicos especializados en las temáticas contempladas (congresos, jornadas, etc.).

A partir de estas actividades en el territorio, se propone a los alumnos un trabajo en instancias áulicas donde se analizan conjuntamente los emergentes y los interrogantes surgidos del encuentro con la comunidad. En estas instancias se recuperan y retoman los problemas identificados en el barrio, se consideran los recursos con que cuentan las organizaciones del sistema de salud, se discuten las distintas perspectivas en torno al abordaje de la problemática, se busca relacionar lo observado con lo leído y estudiado del material propio de la asignatura, etc. Los problemas no son definidos a priori por los docentes sino que surgen de las primeras inserciones de los estudiantes en el campo y las clases se configuran en espacios de discusión y análisis de problemas y sus estrategias de abordaje.

El cursado de la asignatura se divide en tres etapas. La primera se centra principalmente en el relevamiento de datos e información sobre las organizaciones y comunidades. La segunda etapa avanza en el análisis de esas organizaciones, la problematización de lo observado en ellas y la definición de problemas factibles de ser abordados. En la tercera etapa se definen las estrategias de abordaje de los problemas, elaborando un proyecto que contempla los recursos necesarios y disponibles. La participación de las organizaciones y comunidades en este proceso es diferente en cada caso, aunque se intenta sostener un diálogo con ellas a lo largo de las tres etapas.

Para guiar a los estudiantes en el desarrollo del trabajo, los docentes elaboraron diferentes guías: para las visitas a las organizaciones, para la realización de encuestas y entrevistas con actores de la comunidad y/o de las organizaciones, para la confección de proyectos, etcétera.

Luego de cada etapa, los grupos de estudiantes deben presentar un informe del trabajo realizado. Los informes son evaluados por el equipo docente y luego se hacen devoluciones particulares a cada grupo con el objetivo de abrir debates y volver a pensar sobre lo que se plantea.
Los criterios y aspectos a tener en cuenta para las evaluaciones de estos informes, a su vez, se explicitan a los estudiantes mediante guías y rúbricas. Algunos de ellos son: caracterización de la organización y de los problemas de salud de la población o de la organización; enunciación de los criterios de la elección del problema; análisis de las consecuencias y las causas del problema, etcétera. Finalmente, para evaluar la metodología desarrollada, la cátedra aplica encuestas a los alumnos participantes en dos momentos: a la mitad y al final de su cursado. Algunas de las preguntas que conforman la encuesta de final de cursado son las siguientes:

- ¿Qué pudo conocer y aprender durante el cursado de la asignatura?

- ¿En qué medida pudo alcanzar los propósitos que se pretenden alcanzar en la misma?

- El tiempo que tuvo que dedicarle a las actividades propuestas por la cátedra, ¿cómo lo calificaría?

- ¿Había participado antes de actividades de extensión universitaria?

- Con relación a la metodología de aprendizaje basado en proyectos, ¿cree que es una opción de enseñanza-aprendizaje adecuada?

- ¿Había participado antes en la implementación de un proyecto? - ¿Cuáles fueron los obstáculos que encontró en el desarrollo del proyecto?

- ¿El cursado de la asignatura demandó de trabajo en grupo para planificar tareas? ¿Para coordinar actividades? ¿Para debatir o discutir alternativas? ¿Para redactar el documento? ¿Para buscar información?

- ¿Le sirvieron las devoluciones del equipo de cátedra para repensar el trabajo? ¿Por qué?

- La interacción con los docentes, ¿en qué medida contribuyó con el desarrollo de su trabajo?

- ¿Qué aspectos tendría en cuenta en la implementación de un nuevo proyecto?

- Aspectos que valora como positivos del equipo de cátedra, aspectos negativos que deberían mejorarse en próximas cursadas. - ¿Cree que es relevante el aprendizaje de la Salud Pública en la carrera de Bioingeniería?

- ¿De qué manera fueron cubiertas las expectativas que tenía cuando decidió cursar la asignatura? ¿Por qué?

\section{Resultados}

Desde el año 2014 los estudiantes abordan en el marco de la asignatura problemáticas sociosanitarias diversas que surgen de sus primeras inserciones en el terreno y de los análisis que realizan junto a los docentes. 


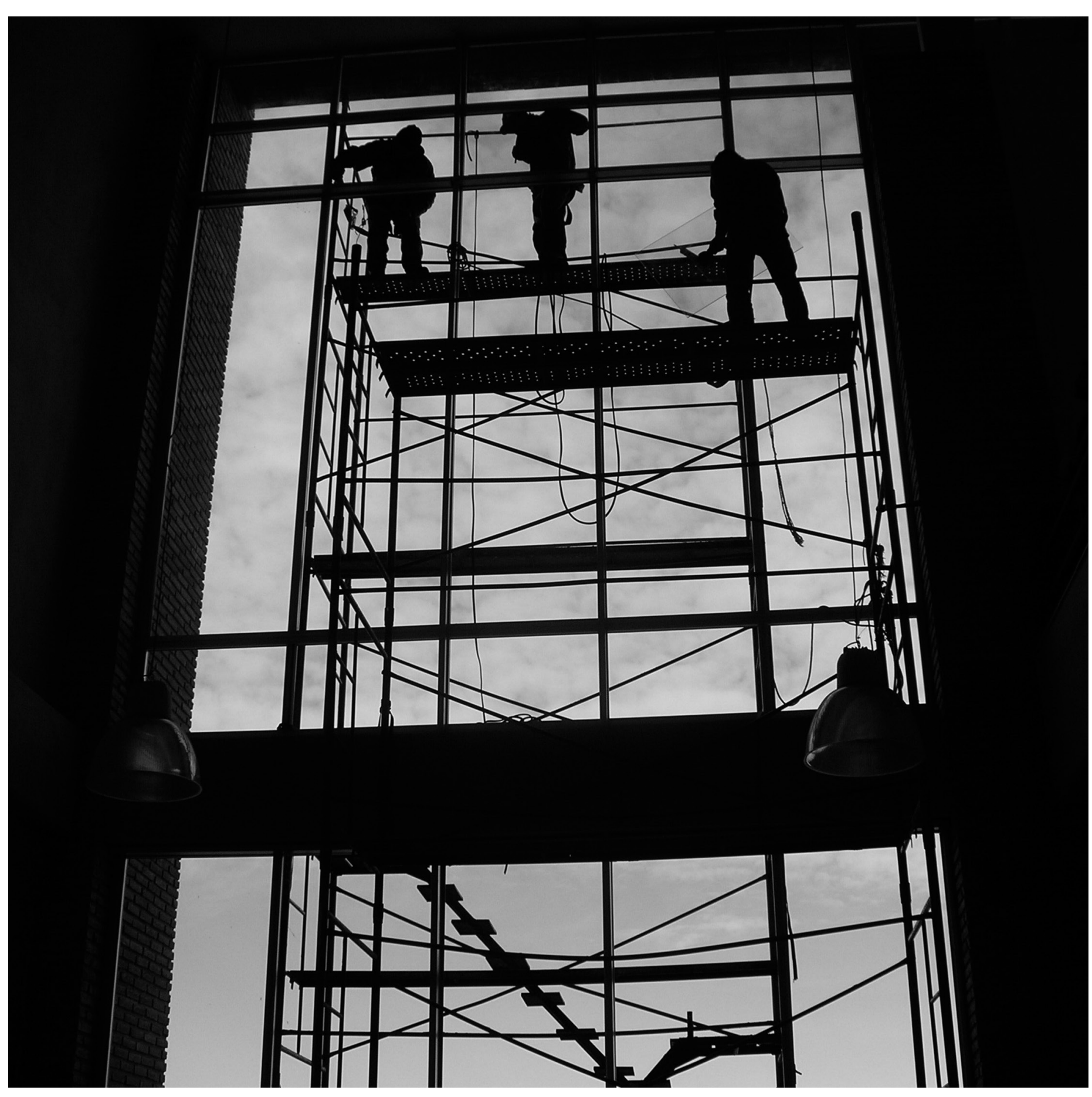

(c) Laura Hormaeche 
A partir de ello se han formulado proyectos que proponen intervenciones diversas tales como: actividades de formación de jóvenes preventores para abordar la problemática de las adicciones, charlas informativas para familiares y para docentes de las escuelas de los barrios involucrados, actividades deportivas y culturales para los niños y jóvenes, implementación de un sistema de información basado en software libre para un Centro de Atención Primaria de la Salud, definición de estrategias para mejorar el estado de salud de la población universitaria en la ciudad de Oro Verde.

Por su parte, algunas de las organizaciones sociales con las cuales se ha trabajado desde el año 2014 en los diferentes cuatrimestres fueron: Centro de Salud H. D’Angelo (Paraná), Red Creer (Paraná), Centro Huella (HESM) (Paraná), Secretaria de Lucha contra las Adicciones (Paraná), Programa Provincial de Alcoholismo y otras Adicciones (Entre Ríos), Escuela de Gestión Social Pablo de Tarso (Paraná), Comisión Vecinal del barrio Padre Kolbe (Paraná); Instituto Provincial de Redes Territoriales de Prevención de Adicciones (Entre Ríos), Servicio de Prevención y Atención a Usuarios de Drogas y Alcohol (Paraná), Grupo Institucional de Adicciones del Hospital Escuela de Salud Mental (Paraná), Centro de Atención Primaria de la Salud "Eva Perón" (Strobel), y Programa de Municipios y comunidades Saludables del Municipio (Oro Verde). La cantidad de estudiantes que cursaron la asignatura en los últimos tres cuatrimestres $\left(2^{\circ}\right.$ cuatrimestre $2014,1^{\circ}$ y $2^{\circ}$ cuatrimestres 2015), en promedio, fue de 13.

A continuación se muestran algunos resultados de la encuesta de final de cursado implementada con alumnos de dichos cuatrimestres que se consideran significativos en función del tema abordado en este artículo.

La mayoría de los estudiantes afirma que antes de esta experiencia no había participado de actividades de extensión universitaria (63\% en 2014; $80 \%$ en $20151^{\circ} \mathrm{C}$; $95 \%$ en $20152^{\circ} \mathrm{C}$ ).

Además, la totalidad de los estudiantes de los tres cursados opina que la opción por la metodología didáctica de ABP es adecuada. Y también en los tres grupos la mayoría considera que dicha metodología ha requerido de un importante trabajo grupal en tareas como las siguientes: planificación y coordinación de actividades, discusión de alternativas y elaboración de informes (más del 74 \% en todos los casos).

Son particularmente significativas, en relación con la temática del presente trabajo, las respuestas a la pregunta: “¿Qué pudieron conocer y aprender durante el cursado de la asignatura?" Y la misma es de respuesta abierta. Seguidamente, se reproducen algunas de estas respuestas correspondientes a los tres cursados mencionados:

- "Lo que pude aprender durante el cursado fue la organización de los distintos niveles de la salud. Creo que fueron muy gratificantes las diversas visitas porque se pudo interactuar con los participantes de todas las áreas y de todos los niveles".
- "Conocer en forma más certera y cercana el sistema público de salud."

- "Una visión realista del sistema de salud, incluidas sus relaciones políticas."

- "Pude conocer el rol de los profesionales que trabajan en las instituciones de salud, su trabajo en grupos interdisciplinarios, su visión de las problemáticas técnicas, sanitarias y políticas."

- "Pude conocer sobre organización de los sistemas de salud de Entre Ríos a través de hablar con múltiples actores y aprender sobre cómo esos actores toman en cuenta, o no, las necesidades de la población al realizar su trabajo."

- "Pude conocer centros de salud que no sabía que existían, ver la realidad en la que trabajan, conocer gente realmente comprometida y con ganas de mejorar las cosas."

- "Instituciones y organizaciones de salud en las cuales pude conocer cómo tratan una problemática como el consumo de drogas."

- "Principalmente crear un ojo crítico o 'curioso' a la hora de analizar un centro de salud. También conocí las adversidades con las que uno se encuentra a la hora de trabajar en un centro de salud."

- "Pude conocer lugares que no conocía y realidades que sabía que existían pero no las conocía. Aprendí que la organización y las ganas de trabajar son claves a la hora de trabajar con centros de salud."

- "Organización de centros de salud; la vida y la realidad en barrios carenciados y disponibilidad de recursos."

- "Hay que darle más dedicación a la parte social del proceso salud-enfermedad."

- "Conocer los centros de salud y su funcionamiento fue un aspecto muy positivo, ya que desconocía la existencia de los mismos.

Además siempre es bueno enfrentarse a una realidad distinta a la habitual y conocer como los trabajadores de los CAPS resuelven muchas cuestiones con recursos escasos."

- "Pude aprender sobre el verdadero nivel de Atención Primaria de la Salud. Se conoció una realidad completamente ignorada y en donde un bioingeniero podría llegar a desenvolverse como profesional."

- "Aprendí también lo complejo y obstaculizado que puede llegar a ser trabajar en forma articulada con otras instituciones, en la planificación y desarrollo de distintas actividades."

- "Pude conocer el impacto que tiene una primera impresión. La importancia de las relaciones humanas ante la implementación de una tecnología. La política y su impacto en las actividades concernientes a cualquier actividad pública."

Por su parte, los docentes reconocen que en el desarrollo del cursado aparecen situaciones impensadas o de baja probabilidad de ocurrencia que promueven el pensamiento y la creatividad en la búsqueda de soluciones con los actores sociales, los procesos 


\section{6}

\author{
los estudios realizados desde el campo \\ de la psicología cognitiva han identificado \\ dos grandes deficiencias del modelo \\ tradicional de educación: el "conocimiento \\ frágil" y el "pensamiento pobre"
}

de reflexión que surgen del cotejo de la realidad de un barrio o de una organización con lo que "debería ser" y con lo que "podría ser" promueven el pensamiento, la planificación y la discusión del rol de los profesionales en la sociedad. La metodología también le permite a las organizaciones reflexionar sobre la situación en la que se encuentran y pensar en términos de proyectos o planes de acción. En cuanto a los aspectos a revisar, los docentes observan que, por los tiempos disponibles para el cursado de una asignatura cuatrimestral, los estudiantes no siempre pueden cumplir con cada una de las etapas del ciclo de observación-problematizaciónplanificación-acción-evaluación de las propuestas. Asimismo, consideran que la conformación de los grupos de trabajo de estudiantes y la construcción de un vínculo docentes-alumnos en ocasiones demanda un tiempo superior al de un cuatrimestre de cursado. Y por último señalan que este tipo de experiencias supone un aumento del tiempo previsto en las actividades de los docentes en terreno, lo cual es central si se quiere propiciar una interacción real entre los estudiantes y los diferentes actores sociales.

\section{Discusión y conclusiones}

A partir de los resultados de las encuestas aplicadas se observa que los estudiantes reconocen que las acciones de articulación docencia-extensión son de central importancia para la formación crítica y contextualizada como futuros profesionales ya que las actividades en terreno les generan preguntas que problematizan el conocimiento brindado por la formación académica. Además, las actividades de extensión integradas a la enseñanza y el aprendizaje permiten que los estudiantes valoren la posibilidad de una mirada interdisciplinaria, donde las ciencias sociales confluyen con la ingeniería en el abordaje de un problema sociosanitario determinado así como de un trabajo intersectorial, donde las redes institucionales cobran gran relevancia.

Por otra parte, la formulación de proyectos contribuyó a que los estudiantes pudieran poner en práctica los contenidos de la asignatura vinculados con la planificación de intervenciones sociales en el área de la salud pública. Consecuentemente, el desarrollo de los contenidos de la asignatura no se realiza de manera aislada a los trabajos de campo, sino que los contenidos en sí mismos se convierten en herramientas para contextualizar la problemática en la población, analizar la situación particular del barrio y su población, 
pensar al sistema de salud como parte de la búsqueda de la solución y formular proyectos que intenten cambiar la situación. En la interacción con los trabajadores de salud y los funcionarios, los estudiantes pudieron conocer, entre otras cosas, los lineamientos de la política sanitaria de cada organismo, las actividades que realizan, las dificultades que presentan, la interacción con la red del primer nivel de atención de la salud, los recursos disponibles y los proyectos en marcha.

Tal como planteaban ya los teóricos de la escuela activa de principios del siglo XX (Dewey principalmente), no toda experiencia es educativa, es decir, no se trata del hacer por el hacer mismo. La experiencia presentada aquí puede ser concebida como una experiencia educativa porque supone un hacer con reflexión, relación teoría-práctica, problematización de fenómenos, datos y hechos observados, contraste de perspectivas de análisis, reconocimiento de la complejidad inherente a todo problema real. Los alumnos aprenden al llevar a cabo las actividades en terreno al discutir en clases, elaborar los informes requeridos y diseñar los proyectos. Todo lo cual queda enriquecido además porque se hace en diálogo con otros: otros pares, docentes, profesionales, organizaciones, comunidades, actores sociales.

En este contexto, las prácticas de enseñanza y de extensión integradas se constituyen en un escenario para que los estudiantes puedan participar activamente en el análisis y toma de posiciones respecto de los problemas observados en la realidad, dialogando con diferentes actores sociales en pos del abordaje de los mismos. Se concluye entonces que la metodología implementada favorece el trabajo colaborativo entre estudiantes, docentes y distintos sectores de la comunidad y permite articular las actividades de docencia de la asignatura con el abordaje de problemas concretos y reales de organizaciones del sistema de salud de la provincia de Entre Ríos en una experiencia altamente formativa para los estudiantes.

El ABP puede concebirse como uno de los modos de instrumentar la integración docencia-extensión, transformadora de varias prácticas e ideas pedagógicas en el ámbito de la universidad.

Finalmente, uno de los aspectos más críticos de esta experiencia tiene que ver con que en la universidad los tiempos de la extensión son diferentes de los tiempos académicos, lo cual genera algunas dificultades para su desarrollo tales como las señaladas por los docentes.

\section{Referencias bibliográficas}

Anijovich, R. y Mora, S. (2010). Estrategias de enseñanza: otra mirada al quehacer en el aula. Buenos Aires: Aique.

Bain, K. (2006). Lo que hacen los mejores profesores universitarios. València: Publicacions de la Universitat de València.

Camilloni, A. (2010). La validez de la enseñanza y la evaluación. ¿Todo a todos? En Anijovich, R. (Comp.), La evaluación significativa. Buenos Aires: Paidós.

Camilloni, A. (2013). La inclusión de la educación experiencial en el currículo universitario". En Menéndez, G. y otros, Integración docencia y extensión. Otra forma de aprender y de enseñar. Santa Fe: Ediciones UNL.

Cecchi, N. (2015). La Universidad que tenemos y la que deberíamos ser: una Universidad socialmente comprometida. Medina, J.M. (Comp.), VI Congreso Nacional de Extensión Universitaria. Rosario: UNR Editora.

Instituto Tecnológico y de Estudios Superiores de Monterrey (ITESM) (2000).

Dirección de Investigación y Desarrollo Educativo. Vicerrectoría Académica: "Las estrategias y técnicas didácticas en el rediseño". Cap. "El método de proyectos como técnica didáctica".

Macchiarola, V. (2010). Incorporación de prácticas Socio-comunitarias al currículo en la Universidad Nacional De Río Cuarto. IV Congreso Nacional de Extensión Universitaria, Universidad Nacional de Cuyo. Extraído de http://www.rexuni.edu. ar/sites/default/files/Viviana_Macchiarola.pdf

Mastache, A. (2007). Formar personas competentes. Buenos Aires: Novedades Educativas.

Perkins, D. (1997). La escuela inteligente. Barcelona: Gedisa.

Ramírez G.; Gantus D. (2012). Documento preliminar de discusión: "Jerarquización de la extensión en la UNER. Apuntes para pensar la curricularización de la Extensión en contexto". Disponible en: www.extension.uner.edu.ar/adjuntos/documentos/CurriculizacionExtension.doc

Resnick, L. y Klopfer, L. (Comps.) (1996). Curriculum y cognición. Buenos Aires: Aique.

Una experiencia de... I Sassetti, F.; Perassi, M.; Kadur, D. y Billordo, J. I 